\title{
Comparison of remotely-sensed and modeled soil moisture using CLM4.0 with in situ measurements in the central Tibetan Plateau area
}

\author{
Shaobo Sun ${ }^{\mathrm{a}, \mathrm{b}}$, Baozhang Chen ${ }^{\mathrm{a}, \mathrm{c}, \mathrm{d}^{*}, \text { Jing Chen }^{\mathrm{e}} \text {, Mingliang Che }}{ }^{\mathrm{a}, \mathrm{b}}$, Huifang Zhang ${ }^{\text {a }}$ \\ ${ }^{a}$ State Key Laboratory of Resources and Environment Information System, Institute of Geographic Sciences and
}

Natural Resources Research, Chinese Academy of Sciences, No. 11A, Datun Road,

Chaoyang District, Beijing, 100101, China

${ }^{b}$ University of Chinese Academy of Sciences, No. 19A, Yuquan Road, Shijingshan District, Beijing 100049, China

${ }^{c}$ School of Environment Science and Spatial Informatics, China University of Mining and Technology,

No. 1, Daxue Road, Quanshan District, Xuzhou, 221116, China

${ }^{d}$ Jiangsu Center for Collaborative Innovation in Geographical Information Resource Development and Application, No. 1, Wenyuan Road, Qixia District, Nanjing, 210023, China

${ }^{e}$ Beijing Meteorological Bureau, No. 44, Zizhuyuan Road, Haidian District, Beijing 100089, China

* Corresponding author Baozhang Chen at: State Key Laboratory of Resources and Environment Information System, Institute of Geographic Sciences and Natural Resources Research, Chinese Academy of Sciences, 11A, Datun Road, Chaoyang District, Beijing, 100101, China.

Tel: +861064889574

E-mail address: baozhang.chen@igsnrr.ac.cn (B. Chen).

\begin{abstract}
Remotely sensed and modeled soil moisture products are widely used in hydro-meteorological, agricultural and other applications. However, both of them need to be validated by ground measurements before they can be used for different applications. Three
\end{abstract} Page 1

(C) 2016. This manuscript version is made available under the Elsevier user license http://www.elsevier.com/open-access/userlicense/1.0/ 
remotely sensed soil moisture products and the simulated soil moisture using the Community Land Model version 4.0 (CLM4.0) were compared and verified with the in situ measurements from a mesoscale soil moisture monitoring network in the central Tibetan Plateau area. These four soil moisture data sets can overall reasonably capture the surface soil moisture dynamics but with considerable biases for some certain soil conditions, such as frozen. The Land Parameter Retrieval Model (LPRM) AMSR-E soil moisture systematically overestimates unfrozen soil moisture and fails to estimates frozen soil, with bias $(B I A S)$ and root mean square error (RMSE) values of $0.11 \mathrm{~m}^{3} \mathrm{~m}^{-3}$ and $0.12 \mathrm{~m}^{3} \mathrm{~m}^{-3}$ in average, respectively. The Japan Aerospace Exploration Agency (JAXA) AMSR-E soil moisture shows better performance than the other two remotely sensed soil moisture, with smaller BIAS and RMSE values (0.017 and $0.096 \mathrm{~m}^{3} \mathrm{~m}^{-3}$ in average, respectively). But it still has a large overestimation bias for unfrozen soil. The AMSR2 data show a similar performance to the JAXA AMSR-E product, but with slight larger BIAS and RMSE $\left(0.024\right.$ and $0.112 \mathrm{~m}^{3} \mathrm{~m}^{-3}$ in average, respectively), and a very large short-term variability during unfrozen periods. The CLM4.0 model was overall able to model multiple-layers' soil moistures well $(R M S E=0.03$ $\left.\mathrm{m}^{3} \mathrm{~m}^{-3}\right)$ but slightly underestimated surface soil moisture $(0-10 \mathrm{~cm})$ and overestimated deeper soil moisture $(20-40 \mathrm{~cm})$ under unfrozen conditions. These comparisons suggest that the LPRM algorithm fails to retrieve soil moisture value in the TP area and the JAXA algorithm needs to be improved for unfrozen soil. For the AMSR2 product, more calibration work and to align it to the AMSR-E sensor are needed . This study also show the potential of using the land surface models (LSMs) for verify the remotely sensed soil moisture products especially for the area with lack of observations available, such as in the Tibetan Plateau area. 


\section{Key words: Soil moisture; Remote sensing; AMSR-E; AMSR2; CLM4.0}

\section{Introduction}

Soil moisture is a key variable in controlling interactions between land surface and the atmosphere (Brocca et al., 2010). Remote sensing and land surface modeling are most common approaches for detecting soil moisture and has been widely applied in hydrology, numerical weather predictions, climatology and agriculture (Koster et al., 2010; Jajarmizadeh et al., 2014; Boisserie and Cocke, 2012; Kim and Wang, 2012; Champagne et al., 2011). However, because of the high spatiotemporal variability of soil moisture, the reliability of these two approaches must be validated and assessed. In the recent years huge efforts have been and are being performed to validate and evaluate these two soil moisture products in many regions (Reichle et al., 2007; Draper et al., 2009; Rüdiger et al., 2009; Jackson et al., 2010; Brocca et al., 2011; Albergel et al., 2012; Su et al., 2013; Coopersmith et al., 2015; Gao et al. 2015; Cho et al., 2015a; Gevaert et al., 2016). Almost all these studies show that the soil moisture derived from remote sensing and land surface models capture serial temporal variations well, but there are still significant uncertainties in different regions, and more evaluation efforts need to be conducted at representative sites with in situ measurements at different scales.

The Advanced Microwave Scanning Radiometer-Earth Observing System (AMSR-E) (http://www.ghcc.msfc.nasa.gov/AMSR/) is one of the most widely used remote sensing sensor for retrieving soil moisture values and has provided continuous products from June 2002 to October 2011. It has now being succeed by the recently launched AMSR2 instrument on the 
JAXA Global Climate Observing Mission-Water satellite (http://www.jaxa.jp/projects/sat/gcom_w/index_e.html). Currently, several AMSR-E soil moisture products by different research groups are available, including the National Aeronautics and Space Administration (NASA) official standard product (Njoku et al., 2003; Njoku and Chan, 2006), the LPRM algorithm product (Owe et al., 2008; de Jeu et al., 2014), the JAXA official standard product (Koike et al., 2004) and the single-channel algorithm (SCA) product (Jackson et al., 1993). Evaluations have shown that these products have varying performance in different regions and under different vegetation conditions (Draper et al., 2009; Jackson et al., 2010; Kim et al., 2015; Zeng et al., 2015), because of the large effects of vegetation and Radio-Frequency Interference (RFI) on the microwave signal recorded by the sensors. For example, Jackson et al. (2010) evaluated four AMSR-E soil moisture data sets in U.S. and found that the JAXA algorithm performs better than the NASA algorithm under light-vegetation conditions, but the NASA algorithm is more reliable for moderate vegetation cover. The LPRM algorithm showed a very large overestimation and retrieval errors. However, several other studies showed that the LPRM soil moisture product may produce relatively high accuracy in capturing the temporal variability in observed soil moisture in several regions, such as Europe (Wagner et al., 2007; Brocca et al., 2011), Australia (Draper et al., 2009) and West Africa (Gruhier et al., 2010), when compared with the NASA AMSR-E soil moisture. The AMSR2 is designed to extend the valuable heritage of AMSR-E and has a similar design with its predecessor. The AMSR2 soil moisture product has been released to the public since May 2013. In the past few years, JAXA has conducted many validation activities in Mongolia, Thailand, Australia, Korean, and the U.S. (Cho et al., 2015b; Parinussa et al., 2015; Kim et al., 2015; Zeng et al., 2015; Wu et al., 2016). As a new passive 
microwave system, it is still necessary to widely validate this product at different spatial scales for a wide range of regions. In addition, inter-comparisons with other independent soil moisture products (such as LSMs based soil moisture) and synergy with the AMSR-E soil moisture products researches are also very important for widespread utilization of the data for scientific research.

LSMs provide another alternative way to derive large-scale and long-term soil moisture products (Georgakakos et al., 2006; Dirmeyer et al., 2006; Zhang et al., 2014). The LSMs have always been off-line applied to produce soil moisture data with high spatial and temporal resolutions, and provide a way to evaluate remote sensing soil moisture products at large-scale (Dirmeyer et al., 2004; Rüdiger et al., 2009; Hain et al., 2011; Albergel et al., 2012; Pan et al., 2014). However, the modeled soil moisture greatly depends on the models' structures, parameterizations and inputs (Lawrence et al., 2011; Guo et al., 2006; Wei et al., 2008; Yang et al., 2009; Singh et al., 2015). For example, Lawrence et al. (2011) reported that the new parameterizations significantly improved the CLM4.0 performance; Swenson et al. (2012) pointed out that the CLM4.0 modeled frozen soil moisture is dry, a likely contribution is excessive movement of liquid water through frozen soil; Guo et al. (2006) and Wei et al. (2008) pointed out that the model forcing largely effect soil moisture simulations; Singh et al., (2015) reported that high-resolution forcing significantly improve the CLM4.0 soil moisture simulations; Yang et al. (2009) and Chen et al. (2012) highlighted that the influences of soil organic carbon (SOC) on LSMs soil moisture simulations should be fully considered in the Tibetan Plateau area, because the high SOC and its large impacts on soil thermal and hydraulic properties. Overall, for the LSMs soil moisture the models' parameterizations and inputs need to be further improved, and wide 
model evaluation efforts are necessary, especially for the cold and high SOC area.

Ground measurements give the most accurate soil moisture estimates and are the indispensable information for validating and evaluating remotely sensed and LSMs modeled soil moisture estimates. Nevertheless, because the scale issues and the high spatial variability and heterogeneity, the ground truth at a satellite pixel or model grid cannot be obtained through measurements at a single station, and measuring networks are necessary to obtain the soil moisture truth at specific spatial scales (Seneviratne et al., 2010; Ochsner et al., 2013). Despite in the past decades attempts have been conducted to establish long-term and large-scale soil moisture observation networks in several regions of the world, as the high economic cost and difficulties in practice only in recent years soil moisture monitoring networks was established on the Tibetan Plateau area (Su et al., 2011; Yang et al., 2013).

The Tibetan Plateau is the highest plateau in the world and located in the central Asia with a mean elevation of over $4000 \mathrm{~m}$ above sea level (ASL) and an area of approximately $2.5 \times 10^{6} \mathrm{~km}^{2}$. The Tibetan Plateau plays an important role in the local and global weather and climate systems(Kang et al., 2010; Yang et al., 2014). To facilitate process studies of soil-vegetation-atmosphere interactions and validate remotely-sensed and modeled soil moistures and temperatures, a mesoscale Soil Moisture and Temperature Monitoring Network on the central Tibetan Plateau (TP-SMTMN) was established (Yang et al., 2013). Using the TP-SMTMN observations Chen et al. (2013) have evaluated the Global Land Data Assimilation System (GLDAS) (Rodell et al., 2004) simulated and the AMSR-E retrieved soil moisture in this area, but no inter-comparisons were conducted and the LSMs in GLDAS are relative earlier versions and the frozen soil parameterizations of which may be insufficient (Xiao et al., 2013). In additional, 
only direct absolute comparisons were conducted in their study, because the mismatch problems of the soil depths and spatial scales for the two soil moisture products these comparisons may be not sufficient. In other studies, Zeng et al. (2015) have also evaluated multiple remotely-sensed and reanalysis soil moisture products in the Tibetan Plateau area. For LSMs approaches, many studies have been conducted for LSMs, such as CLM, Noah, CoLM and others. However, these evaluations were performed with one single station's observations and the representativeness may be not enough (Xia et al., 2011; Li et al., 2012; Lai et al. 2014; Chen et al. 2014; Gao et al., 2015). Singh et al. (2015) reported that a high grid-resolution significantly improves the CLM4.0 model soil moisture simulations. The main purpose of this study is to evaluate the CLM4.0 modeled soil moisture at a high resolution and conduct an inter-comparison with three remote sensing retrieved soil moistures based on the TP-SMTMN measurements. We aim to present a comprehensive validation analysis of the two widely used soil moisture methods (remote sensing and LSMs) in the Third Pole and provide valuable reference information for further improvements of remote sensing algorithms and land surface model parameterization schemes.

The paper is organized into five sections. The methods and data sets used in this paper are described in section 2. In the third section, the results and analyses are introduced. The fourth section presents a discussion of the results. The conclusions and directions for future study are provided in the last section.

\section{Methods and datasets}

\subsection{Study area and the soil moisture monitoring network}

The Soil Moisture and Temperature Monitoring Network on the central Tibetan Plateau (TP-SMTMN) (Fig. 1) is located in the central area of the Tibetan Plateau with an area of 
$100 \times 100 \mathrm{~km}^{2}$, mostly covered by alpine grassland with an average elevation above $4500 \mathrm{~m}$ ASL (Chen et al., 2013). Because of the high elevation, the land-atmosphere interactions in the TP-SMTMN area are strong and soil thawing and freezing occur in approximately May and November, respectively. The TP-SMTMN was completed in June 2012 after three years of construction. It consists of 56 stations continuingly measured soil moisture and temperature at depths of $5 \mathrm{~cm}, 10 \mathrm{~cm}, 20 \mathrm{~cm}$ and $40 \mathrm{~cm}$. More details of the study area and the TP-SMTMN can be found in Yang et al. (2013). To simultaneously evaluate soil moisture estimations derived from AMSR-E and modeled by CLM4.0 and emphasize on the influences of elevation and obtain more representative results, we have redesigned the network (Fig. 1) to comprise a large network (the whole network), medium network (red rectangle), AMSR-E pixel (blue rectangle) and model grid (black rectangle).
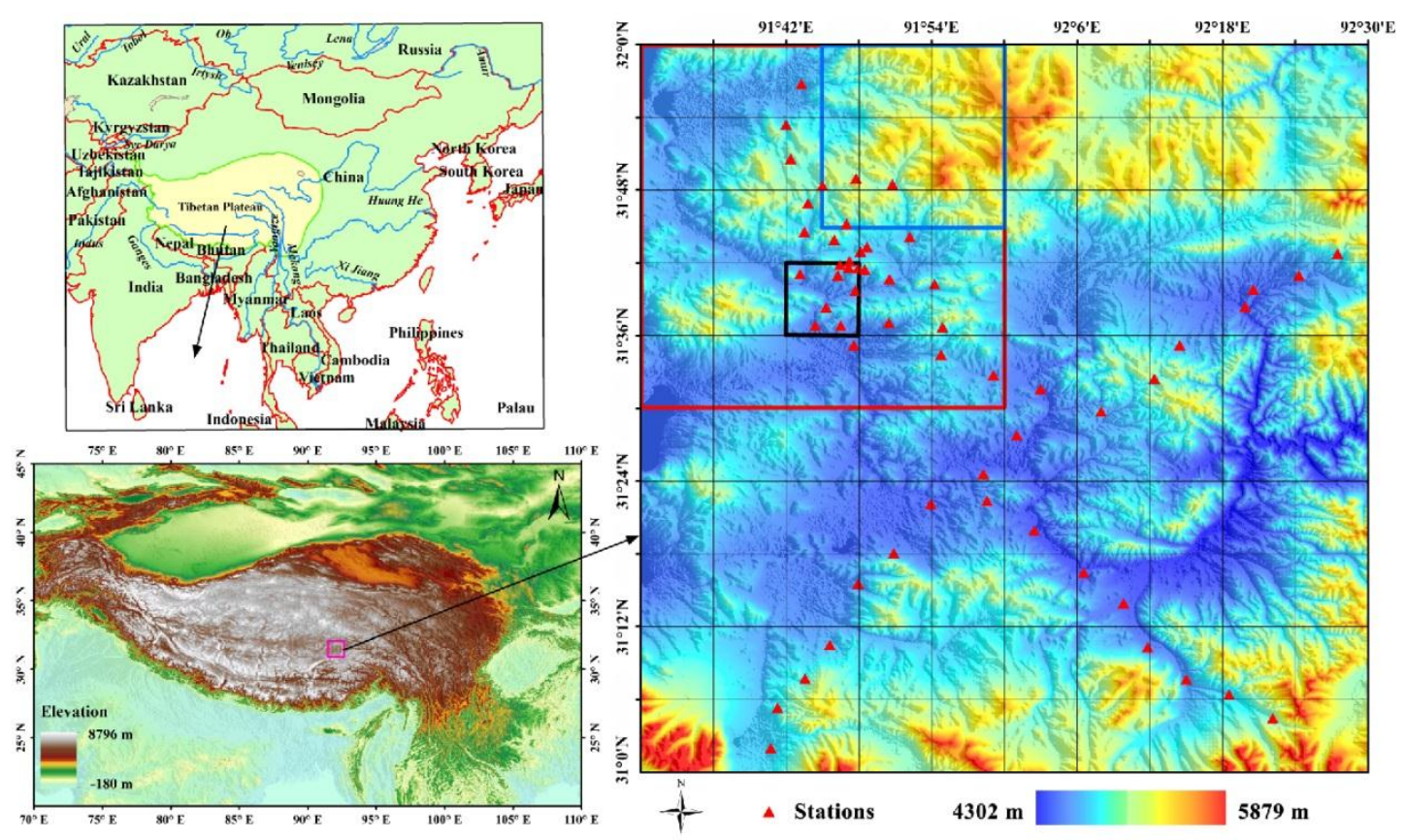

Fig. 1. Location of the soil moisture monitoring network.

\subsection{Remote sensing soil moisture}

\subsubsection{AMSR-E soil moisture}


The AMSR-E sensor onboard the Aqua satellite of NASA has been widely used for monitoring soil moisture. Since May 2002, it has provided passive microwave measurements at six bands (6.9 to $89 \mathrm{GHz})$, with daily ascending (13:30) and descending (01:30) overpasses. As discussed by Draper et al. (2009), Jackson et al. (2010) and Mladenova et al. (2014), several AMSR-E soil moisture products have been developed by different research groups. In this study, the widely used LPRM AMSR-E soil moisture product (AMSR-LPRM) and the JAXA AMSR-E soil moisture products (AMSR-JAXA) were used.

The AMSR-LPRM product was developed by the Vrije Universiteit Amsterdam (VUA) in collaboration with NASA. The algorithm of AMSR-LPRM soil moisture is based on a forward radiative transfer model to retrieve the surface soil moisture and both the C-band and X-band signals are used (Owe et al., 2008). The daily level 3 product $\left(0.25^{\circ}\right.$ and version 2$)$ was used in our study. The AMSR-JAXA soil moisture used is also the daily level 3 product (version 700) which is now released to the public free of charge (https://gcom-w1.jaxa.jp/auth.html). The JAXA algorithm uses a forward radiative transfer scheme to generate brightness temperatures for a range of parameter values and further create lookup tables for estimating soil moisture and vegetation water content (Koike et al., 2004; Fujii et al., 2009).

\subsubsection{AMSR2 soil moisture}

As the successor of the AMSR-E, the AMSR2 onboard the Global Change Observation Mission 1-Water (GCOM-W1) satellite was launched by JAXA in May 2012 (Imaoka et al., 2010). It measures the brightness temperatures at seven different frequencies $(6.925 / 7.3,10.65,18.7,23.8$, 36.5 and $89.0 \mathrm{GHz}$ ) and has almost identical concept to the AMSR-E (Kachi, et al., 2014). The key difference between the AMSR2 and AMSR-2 is that a new 7.3-GHz channel was added to 
AMSR 2 because the RFI signals were frequently found in the $6.925-\mathrm{GHz}$ brightness temperatures of AMSR-E (Wu et al., 2016). The JAXA has developed its AMSR2 soil moisture product (2012.07-) and made it free of charge together with the AMSR-JAXA soil moisture product (https://gcom-w1.jaxa.jp/auth.html). The algorithm of AMSR2 soil moisture is same as that of the AMSR-JAXA. In our study, the AMSR2 level 3 product ( $0.25^{\circ}$ and version 2 ) was used against in situ measurement and compared with the CLM4.0 modeled soil moisture.

\subsection{Model and forcing data}

\subsubsection{Model description}

The LSM used is the Community Land Model version 4.0 (CLM4.0) (Oleson et al., 2010; Lawrence et al., 2011) developed by National Center for Atmospheric Research (NCAR) and was also embedded in the Community Earth System Model version 1.0 (CESM1.0). Although the originally developed CLM was primarily used as a lower boundary condition for atmosphere models and the terrestrial component within the Earth System Model (ESM), the off-line CLM model has also become a useful tool to understand the complex land contributions and responses to climate variability and changes. Compared with previous versions, the CLM4.0 model has several crucial improvements in model parameterization and structure. For example, the soil and hydrology module was significantly improved by using the modified Richards equation; the terrestrial carbon and nitrogen cycles modules were added in. The soil physics of CLM4.0 includes vertically resolved thermal and hydrological diffusion in 10 soil layers down to $3.8 \mathrm{~m}$, with five hydrologically inactive bedrock layers continuing down to $42 \mathrm{~m}$. Energy and water balances are calculated for each tile of CLM4.0 are conserved and integrated by an implicit time-integration scheme. In this study the CLM4.0 model was run off-line and modelled soil 
moisture at the top layers was used.

\subsubsection{Model forcing data}

The forcing data set used in this study was developed by the Data Assimilation and Modeling Center for Tibetan Multi-spheres, Institute of Tibetan Plateau Research, Chinese Academy of Sciences

(ITP-CAS)

(http://dam.itpcas.ac.cn/data/User_Guide_for_China_Meteorological_Forcing_Dataset.htm) (Yang et al., 2010). It currently covers the period of 1979-2012 with a temporal and spatial resolution of every $3 \mathrm{~h}$ and $0.1^{\circ} \times 0.1^{\circ}$ in longitude and latitude. The air temperature, wind speed, atmospheric pressure, specific humidity were produced by merging observations from 740 meteorological stations of China Meteorological Administration (CMA) into the corresponding Princeton meteorological forcing data (Sheffield et al., 2006). The precipitation was constructed by combining observations from the 740 CMA stations, the Tropical Rainfall Measuring Mission (TRMM) 3B42 precipitation products (Huffman et al., 2007), and the Asian Precipitation-Highly Resolved Observational Data Integration Towards Evaluation of the Water Resources project (APHRODITE) precipitation data (Yatagai et al., 2009). The downward shortwave radiation was produced by correcting the Global Energy and Water Cycle Experiment-Surface Radiation Budget shortwave radiation data set (Pinker and Laszlo, 1992) with radiation estimates from meteorological station data. The downward longwave radiation was estimated using Crawford and Duchon's (1999) model method. Several evaluations and applications have shown that this forcing data yielded reliable results in China (Guo and H. Wang, 2013; Zhang et al., 2014). Hence, we selected the ITP-CAS data as the CLM4.0 model forcing data. The model was run at the resolution $\left(0.1^{\circ} \times 0.1^{\circ}\right)$ and a 50 -year spinning-up was conducted before the outputs were used for the 
comparisons.

\subsection{Assessing methods}

In this study, both AMSR-E derived and CLM4.0 simulated soil moisture values were evaluated using the TP-SMTMN observations at different scales. The statistical scores for all the time series comparisons were determined with the mean bias (BIAS, Eq. 1), the root mean square error (RMSE, Eq. 2), and the correlation coefficient (R, Eq. 3).

$$
\begin{gathered}
\text { BIAS }=\frac{1}{n} \sum_{i=1}^{n}\left(S M_{\text {insitu }}-S M\right) \\
R M S E=\sqrt{\frac{1}{n} \sum_{i=1}^{n}\left(S M_{\text {insitu }}-S M\right)^{2}} \\
R=\frac{\sum_{i=1}^{n}\left(S M_{\text {insitu }}-\overline{S M_{\text {insitu }}}\right)(S M-\overline{S M})}{\sqrt{\sum_{i=1}^{n}\left(S M_{\text {insitu }}-\overline{S M_{\text {insitu }}}\right)^{2} \sum_{i=1}^{n}(S M-\overline{S M})^{2}}}
\end{gathered}
$$

where $S M_{\text {insitu }}$ and $S M$ are in situ measurement and soil moisture product (remote sensing or CLM4.0) values, respectively. $\overline{S M_{\text {insitu }}}$ and $\overline{S M}$ are the average values of in situ measurement and soil moisture product, respectively.

In order to eliminate the mismatch problems of the soil depths and spatial scales, normalized anomalies (Ano) were also calculated which are defined as the differences of the time series of the soil moisture subtracted by the average divided by standard deviation $\left(\sigma_{s m}\right)$ for the time series (Gruhier et al., 2008; Choi, 2012) (Eq. 4).

$$
A n o=\frac{\overline{S M}-S M}{\sigma_{s m}}
$$

Additionally, the normalized standard deviation $(S D V)$ was calculated to evaluate all the stations' pixels or grids with a Taylor diagram (Taylor, 2001). The SDV is the ratio of the soil moisture product standard deviation and in situ measurement standard deviation: 


$$
S D V=\frac{\sigma_{\text {product }}}{\sigma_{\text {insitu }}}
$$

where $\sigma_{\text {product }}$ is the standard deviation of the remote sensing or CLM4.0 soil moisture values, and $\sigma_{\text {insitu }}$ is the standard deviation of the in situ measurements. The $S D V$ is displayed as a radial distance in the polar plot, and the correlation with in situ data is displayed as an angle. An ideal modeled result would have a standard deviation ratio of 1.0 and a correlation coefficient of 1.0 (i.e., the reference point on the $x$ axis). The distance to this point represents the centered normalized root mean square difference between the analyzed and in situ data.

\section{Results}

\subsection{Comparison of CLM4.0 modelled soil moisture with in situ observations}

In this section, the absolute values of the CLM4.0 modeled daily soil moisture were compared with the in situ measurements. The comparisons were conducted at three scales: the large network scale (the whole network in Fig. 1), medium network scale (red rectangle) and model grid (black rectangle). In order to match to the in situ observation depths, the top six soil layers $(1.75,4.51$, $9.06,16.55,28.91$ and $49.29 \mathrm{~cm}$ ) of the model outputs were used and linearly interpolated to the observation depths $(5,10,20$ and $40 \mathrm{~cm})$.

The in situ soil moisture series in Figs. 2-5 display a high seasonal variation, ranging from 0.04 to $0.40 \mathrm{~m}^{3} \mathrm{~m}^{-3}$. Soil moisture dropped below $0.2 \mathrm{~m}^{3} \mathrm{~m}^{-3}$ when it began to freeze in November, while it began to rise when soil began to thaw in late April. This suggests that the soil freeze-thaw cycle is strong in this area. The modeled and in situ measured soil moisture series at the three network scales showed similar variations, indicating that the spatial variability of soil moisture is relatively small and a robust evaluation can be obtained. Overall, the modeled and in situ measured soil moisture is highly correlated in terms of their temporal dynamics despite some 
biases for frozen and unfrozen soil. For example, Fig. 2 shows that the model distinctly overestimates top layer soil moisture values when soil was frozen and the liquid water content was small $\left(<0.08 \mathrm{~m}^{3} \mathrm{~m}^{-3}\right)$, and underestimates soil moisture when soil was frozen but the liquid water content above $0.1 \mathrm{~m}^{3} \mathrm{~m}^{-3}$; For unfrozen soil, the model slightly underestimated the top layer soil moisture. Fig. 3 presents the comparisons between the second soil layers $(10 \mathrm{~cm})$ of CLM4.0 modeled and in situ measurements. It is similar to Fig. 2 that the model can capture unfrozen soil moisture dynamics well despite a slightly dry bias. For frozen soil, the model simulated soil moisture well in 2013, but significantly underestimated in 2014. Figs. 4 \& 5 display comparisons of the deeper soil layers (20 and $40 \mathrm{~cm}$ ). The time series show that the model slightly overestimated soil moisture during unfrozen periods and also had significant uncertainties during frozen periods. In general, the comparisons suggest that forced with high quality atmospheric forcing data the model can reflect multiple-layers' soil moisture dynamics well despite some uncertainties such as slight biases for unfrozen soil and uncertainties for frozen soil. 


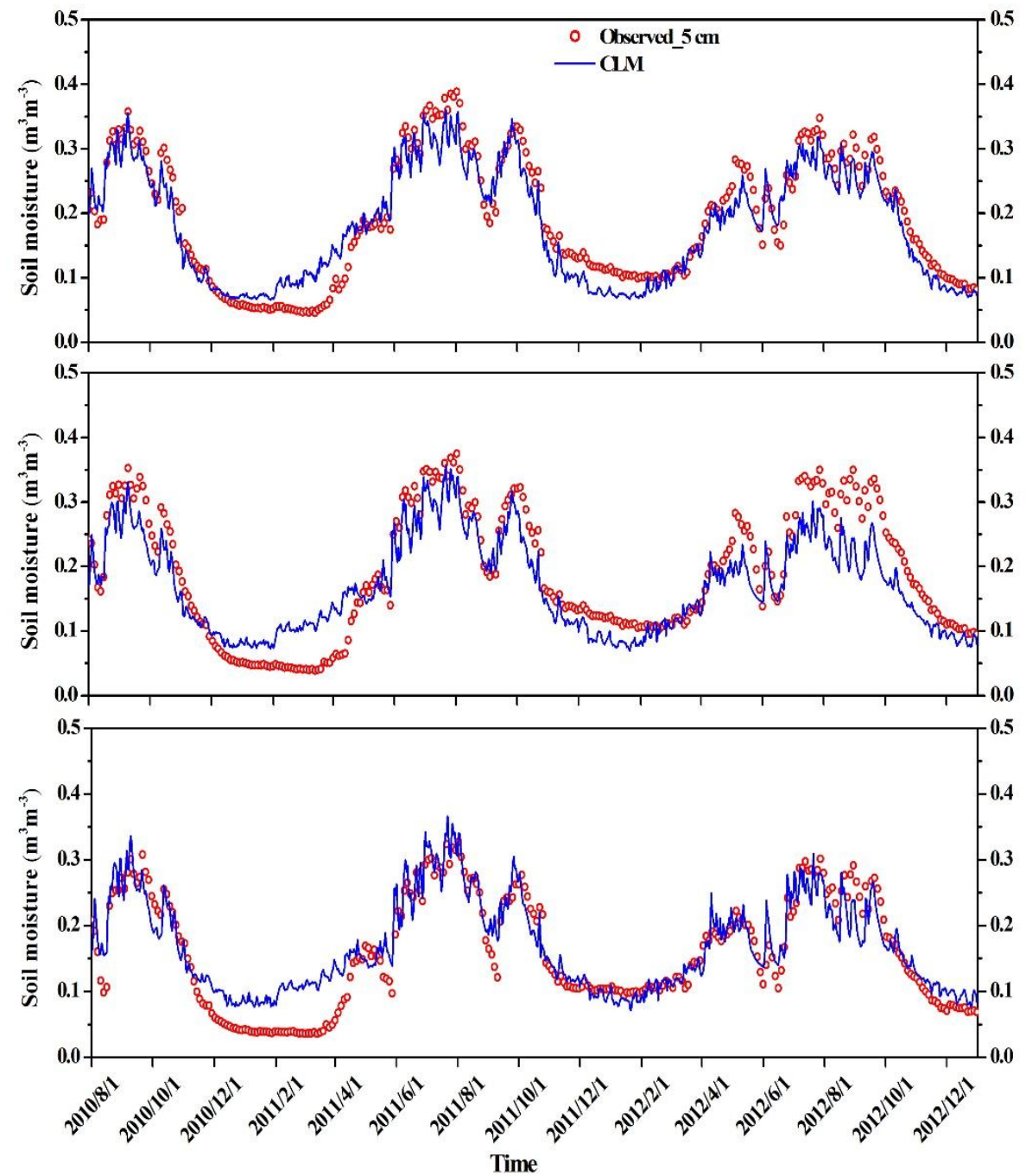

Fig. 2. Soil moisture data; in situ observed and CLM4.0 modeled at $5 \mathrm{~cm}$ depth, from 1 August 2010 to December 2012 at three scales. a. large network; b. medium network; and c. model grid. 


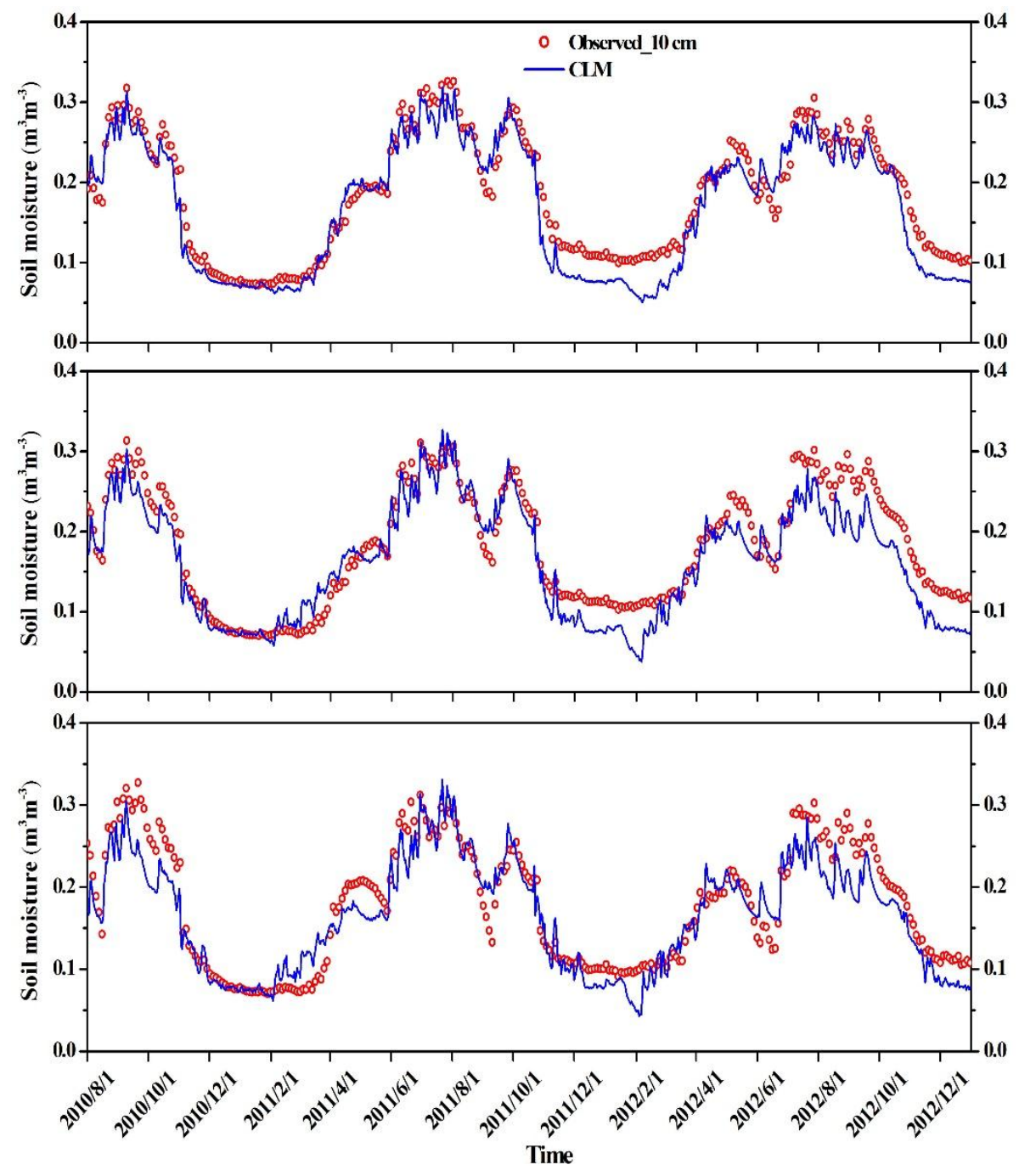

Fig. 3. Same as Fig.2 but for $10 \mathrm{~cm}$ depth. 


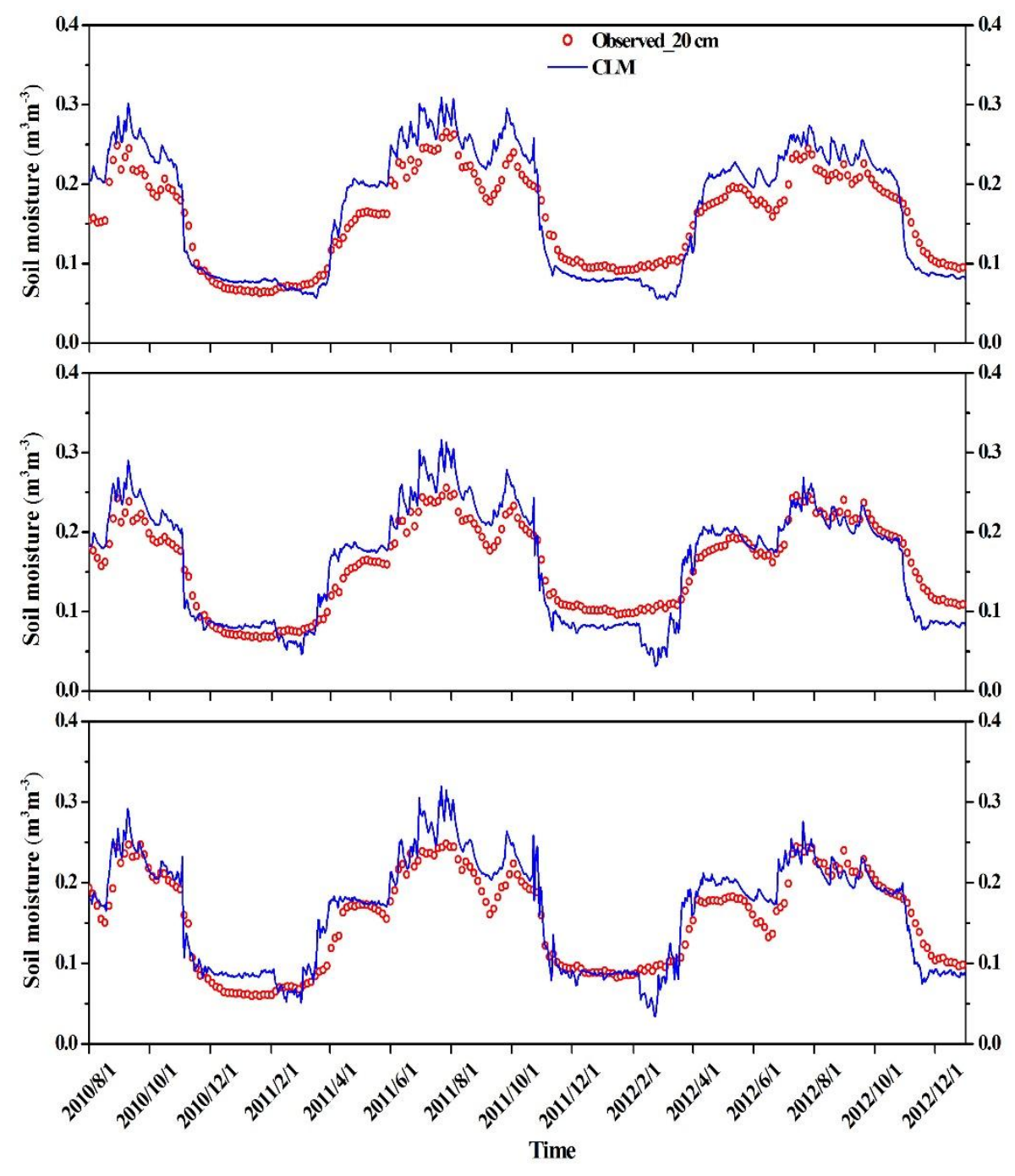

Fig. 4. Same as Fig.2 but for $20 \mathrm{~cm}$ depth. 


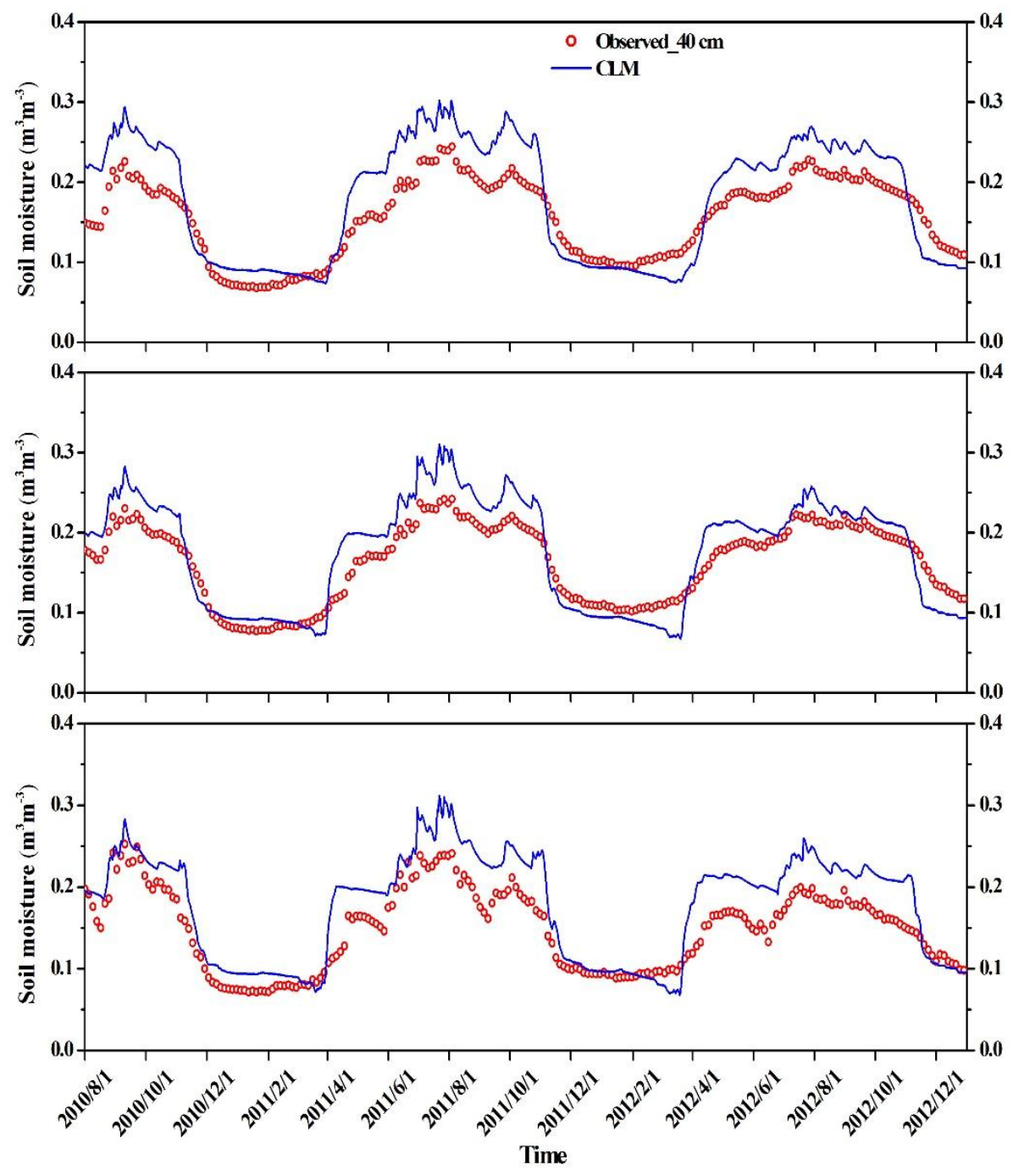

Fig. 5. Same as Fig.2 but for $40 \mathrm{~cm}$ depth.

Table 1 presents the results of the comparisons in terms of BIAS, RMSE, and R. The BIAS values range from 0.004 to $0.03 \mathrm{~m}^{3} \mathrm{~m}^{-3}$ and in average of $-0.007,-0.015,0.009$ and $0.022 \mathrm{~m}^{3} \mathrm{~m}^{-3}$ for 5, 10, 20 and $40 \mathrm{~cm}$ depths, respectively. The negative values indicate that the CLM4.0 model slightly underestimates soil moisture at the surface soil layers $(<10 \mathrm{~cm})$, and the positive bias values suggest that the model overestimated soil moisture value for deep soil layers ( $>20 \mathrm{~cm}$ ). In average, RMSE values are $0.037,0.029,0.028$ and $0.036 \mathrm{~m}^{3} \mathrm{~m}^{-3}$ at soil depths of 5, 10, 20 and 40 $\mathrm{cm}$, respectively. These results seem acceptable for most applications. For all the soil layers, good correlations were obtained with a mean value of 0.94 . 
Table 1 Statistics of CLM4.0 modeled soil moisture for TP-SMTMN (Units: $\mathrm{m}^{3} \mathrm{~m}^{-3}$ )

\begin{tabular}{ccccc}
\hline \multirow{2}{*}{ Networks } & Depths & \multicolumn{3}{c}{ Statistic scores } \\
\cline { 3 - 5 } Large & $(\mathrm{cm})$ & $B I A S$ & $R M S E$ & $R$ \\
& 5 & -0.0101 & 0.0318 & 0.9528 \\
& 10 & -0.0143 & 0.0253 & 0.9652 \\
& 20 & 0.0138 & 0.0313 & 0.9601 \\
Medium & 40 & 0.0236 & 0.0398 & 0.9423 \\
& 5 & -0.0203 & 0.0461 & 0.9239 \\
& 10 & -0.0180 & 0.0308 & 0.9393 \\
Single grid & 20 & 0.0041 & 0.0269 & 0.9404 \\
& 40 & 0.0134 & 0.0291 & 0.9509 \\
& 5 & 0.0102 & 0.0338 & 0.9259 \\
& 10 & -0.0126 & 0.0302 & 0.9288 \\
& 20 & 0.0103 & 0.0249 & 0.9422 \\
& 40 & 0.0296 & 0.0380 & 0.9459 \\
\hline
\end{tabular}

Furthermore, Fig. 6 presents four Taylor diagrams illustrating the statistics of the comparisons between measured and the modelled soil moisture values, corresponding to the soil depths of 5,10 , 20 and $40 \mathrm{~cm}$. Each station (Fig. 1) of the TP-SMTMN was presented as a point in the diagrams. Overall, the modeled soil moistures at four depths had a similar $R$ value of 0.9 , but the shallow layers perform better than deep layers with $S D V s$ closer to 1 and greater $R$ values. Fig. $6 \mathrm{a}$ and b show that most of the $R$ values at the depths of 5 and $10 \mathrm{~cm}$ were between 0.85 and 0.95 , and the $S D V$ values are also well-distributed over the REF line. As $S D V$ is the ratio between the standard deviation of soil moisture products and in situ measurements (Eq.1). These results indicate that the variabilities of modeled and observed soil moistures were similar for the surface layers. While for deeper layers (Fig. 6c and d), although most of the correlation coefficients values were also higher than 0.85 , the $S D V$ values were generally higher than 1.0 (i.e., the REF line) and had dispersive distributions. This result is corresponding to the time series plot figures (Figs. $4 \& 5$ ), suggesting that the variability of the CLM4.0 modeled soil moistures for deep layers are higher than in situ data. 

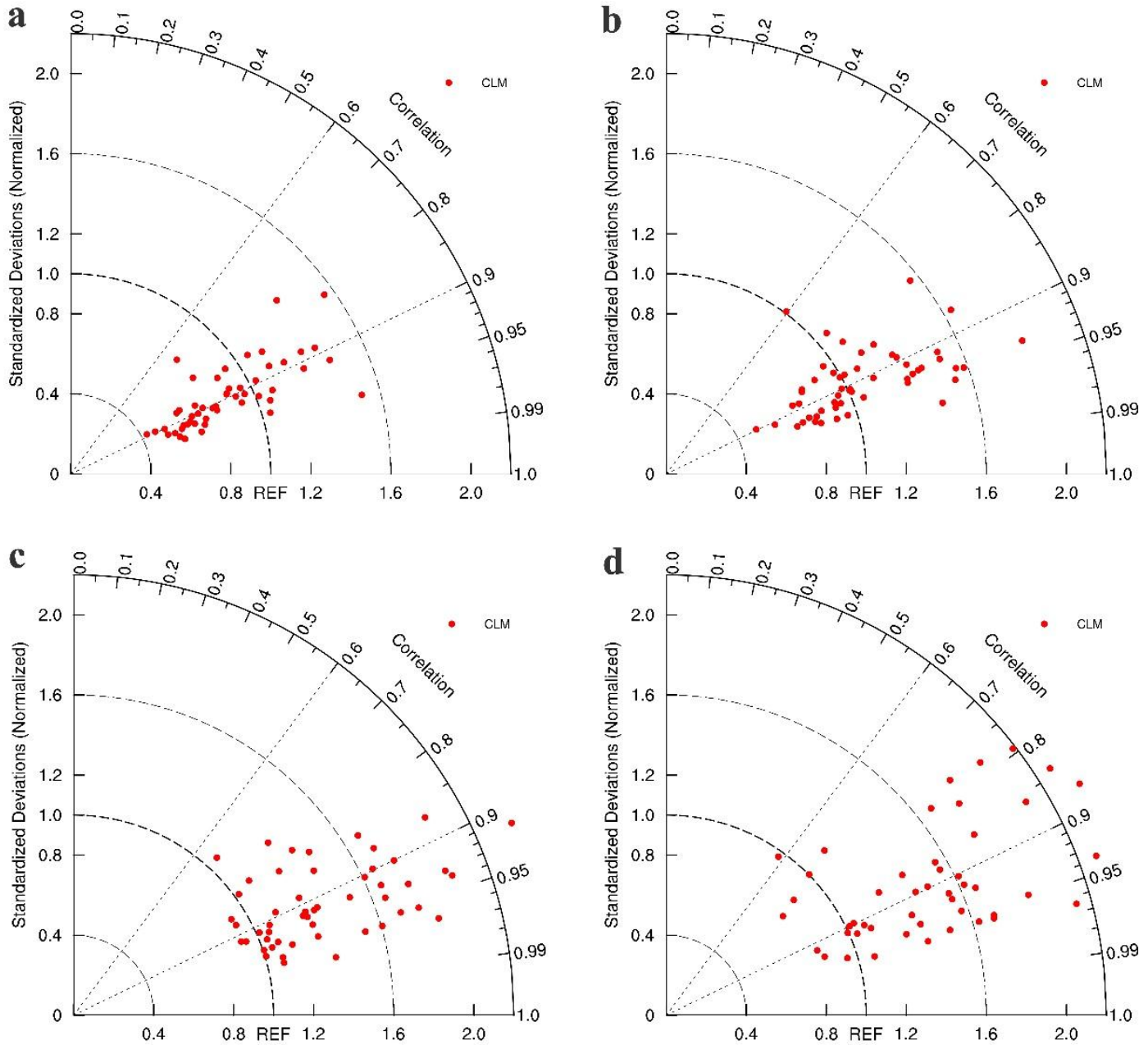

Fig. 6. Taylor diagrams of the comparisons between CLM4.0 modeled and in situ measured soil moistures at different depths a. $5 \mathrm{~cm}$; b. $10 \mathrm{~cm}$; c. $20 \mathrm{~cm}$; and d. $40 \mathrm{~cm}$ depth.

\subsection{Inter-comparisons between the remote sensing and CLM4.0 soil moisture}

\section{estimates}

The modeled and in situ data used here are the satellite passing time data (ascending and descending time), and the time series comparisons were conducted at the large network scale, medium network scale and AMSR-E pixel scale (blue rectangle in Fig. 1).

In first step, the absolute values of soil moisture were compared. Overall, all the remotely sensed and modeled soil moisture products reflect soil moisture seasonal dynamics well for both ascending and descending orbits (Figs. 7 \& 8). However, there are significant biases for each soil 
moisture product. The AMSR-LPRM distinctively overestimates soil moisture values under unfrozen condition and fails to estimate frozen soil moisture for both the C and X-band data. The AMSR-JAXA performs best for deriving frozen soil moisture values, when compared with the other two remotely sensed soil moisture data sets. Nevertheless, it also significantly overestimates soil moisture values during unfrozen periods. It should be noticed that both the AMSR-JAXA and AMSR2 soil moisture show larger short-term variability than AMSR-LPRM for unfrozen soil. This result indicates that the JAXA algorithm is more sensitive to soil water content changes than LPRM algorithm. Because in situ measurements cover August 2010 to December 2012 and the AMSR2 soil moisture product is since July 2012, the AMSR2 soil moisture used in our study is from July 2012 to December 2012. Although this temporal coverage is short, it is still shown that the AMSR2 clearly reflects soil moisture seasonal changes. Particularly, it should be noticed that the AMSR2 data have a very large short-term variability for unfrozen periods and underestimates soil moisture during frozen-thaw periods. These results indicate that as the successor of AMSR-E, more researches are needed to calibrate the AMSR2 soil moisture product and further align it to the AMSR-E sensor (Parinussa et al., 2015). Compared to the remote sensing soil moisture, the modeled soil moisture shows better performance, with relative small uncertainties. 


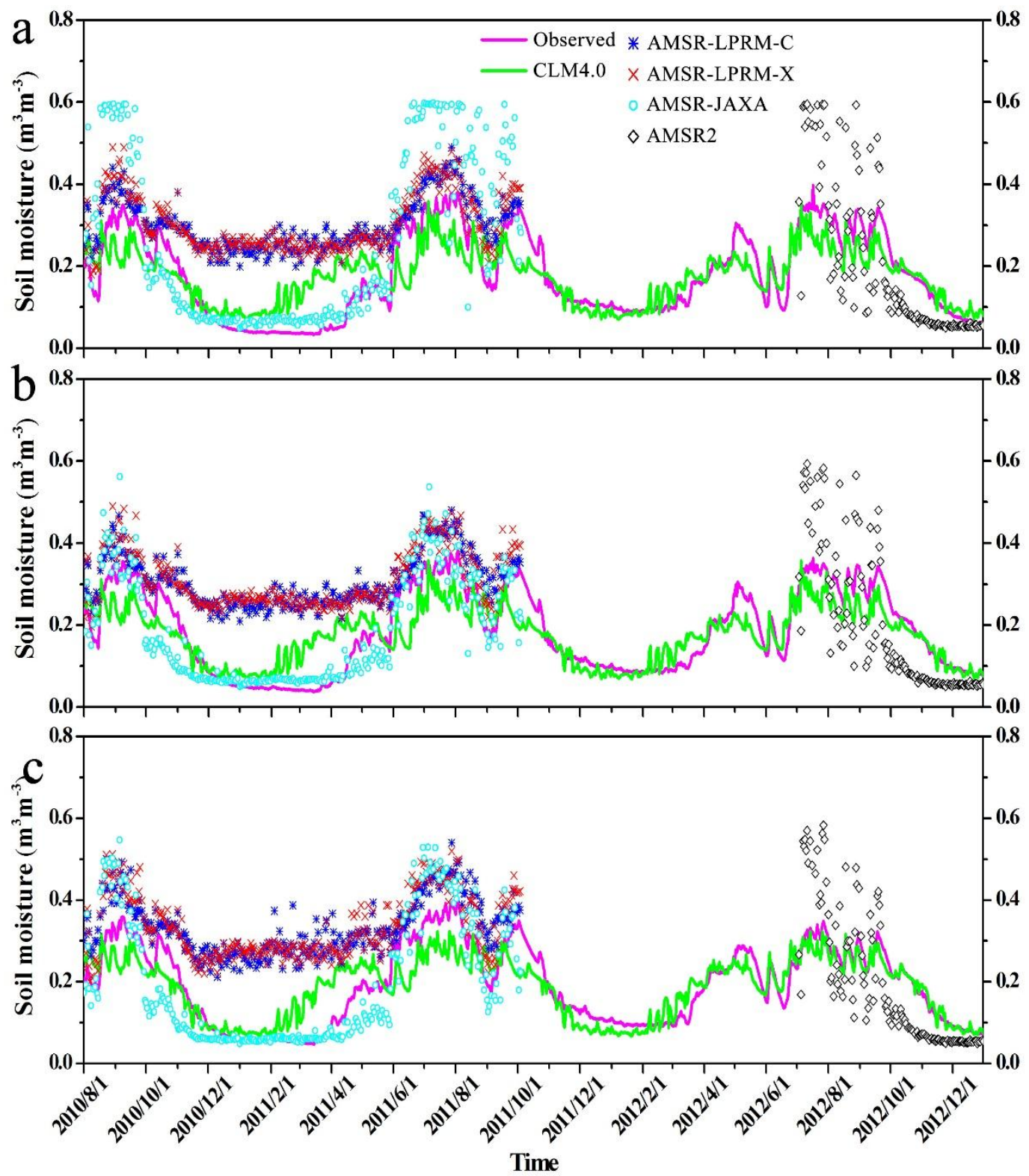

Fig. 7. Soil moisture time series at three scales: in situ measurements, the CLM4.0 model, the Cand X-band of AMSR-LPRM (AMSR-LPRM-C and AMSR-LPRM-X), the AMSR-JAXA and AMSR2 for ascending orbit. a. AMSR-E pixel; b. medium network; and c. large network 


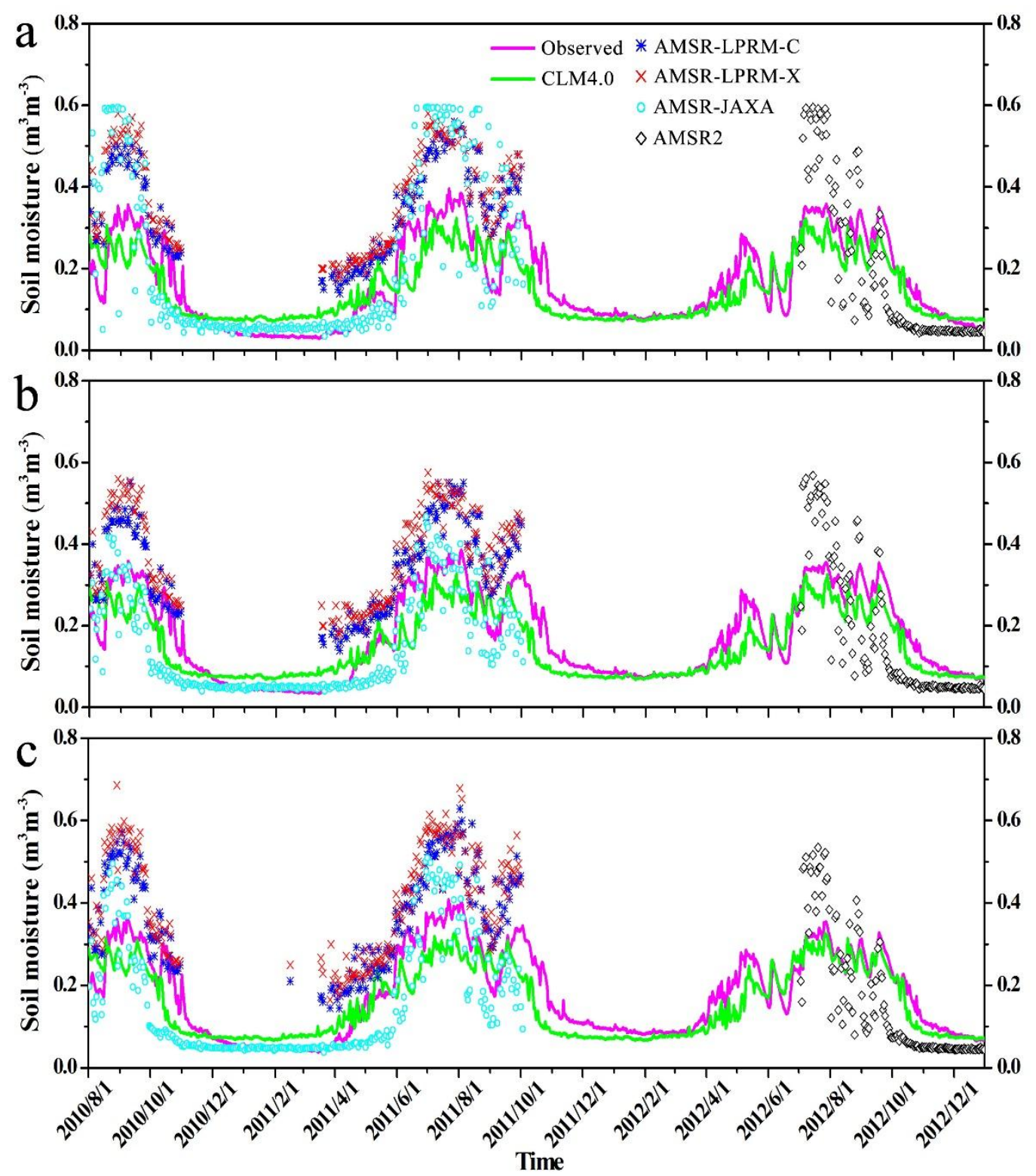

Fig. 8. Same as Fig.7, but for descending orbit.

Table 2 presents the error metrics of the soil moisture products. All the statistic values are the average of that of the three scales. For the AMSR-E-LPRM, because detecting frozen soil moisture is beyond its capacity, only the data during unfrozen periods were calculated. As it is shown, there are not large differences between the $\mathrm{C}$ and $\mathrm{X}$-band data, because the biomass, air mass and air moisture are low and the RFI is rare in this study area. Although high $R$ values (> $0.83)$ were obtained, the mean values of $\operatorname{BIAS}\left(0.11 \mathrm{~m}^{3} \mathrm{~m}^{-3}\right)$ and $R M S E\left(0.12 \mathrm{~m}^{3} \mathrm{~m}^{-3}\right)$ are largest, when compared with other soil moisture. These results suggest that the AMSR-LPRM 
systematically overestimate soil moisture values under unfrozen condition. Compared with the AMSR-LPRM and AMSR2 data, the AMSR-JAXA shows the best performance with average BIAS, RMSE and $R$ values of $0.017 \mathrm{~m}^{3} \mathrm{~m}^{-3}, 0.096 \mathrm{~m}^{3} \mathrm{~m}^{-3}$, and 0.85 , respectively. However, the RMSE values indicate that the uncertainties are still large. The AMSR2 performs slight poorer than the AMSR-JAXA data, although their algorithms are same. Because of the limited temporal coverage, the comparisons for AMSR2 may be insufficient and more evaluation efforts are needed. The average $R$ value of CLM4.0 simulation is 0.85 , and the BIAS and RMSE values (0.013 and $0.057 \mathrm{~m}^{3} \mathrm{~m}^{-3}$ ) are much lower than the three remotely sensed products. These results suggest that the model outperforms the remotely sensed soil moisture in this study area. In addition, Table 2 also shows that there are not large differences between the ascending and descending data for both CLM4.0 and remotely sensed soil moisture.

Table 2 Statistics of the remote sensing and CLM4.0 soil moistures for TP-SMTMN

\begin{tabular}{lcccccc}
\hline \multirow{2}{*}{ Products } & \multicolumn{3}{c}{ Ascending } & \multicolumn{3}{c}{ Descending } \\
\cline { 2 - 7 } & $B I A S$ & $R M S E$ & $R$ & $B I A S$ & $R M S E$ & $R$ \\
AMSR-C & 0.0907 & 0.1028 & 0.8312 & 0.1171 & 0.1290 & 0.8937 \\
AMSR-X & 0.0935 & 0.1035 & 0.8508 & 0.1465 & 0.1585 & 0.8854 \\
AMSR-JAXA & 0.0249 & 0.0937 & 0.8767 & -0.0081 & 0.0981 & 0.8209 \\
AMSR2 & -0.0170 & 0.1112 & 0.8207 & -0.0313 & 0.1141 & 0.7809 \\
CLM4.0 & -0.0065 & 0.0591 & 0.8179 & -0.0200 & 0.0540 & 0.8852 \\
\hline
\end{tabular}

Fig. 9 shows two Taylor diagrams of the comparisons between in situ measurements and the remote sensing soil moistures. The panels $a$ and $b$ correspond to the ascending and descending orbits data, respectively. For the AMSR-LPRM, the two orbits data have a similar $R$ range (from 0.7 to 0.9 ), but a distinctly different $S D V$ s distribution. Most Ascending $R$ values of the points are above 0.8 and $S D V$ s values below the 1.0. This result indicates that the ascending AMSR-LPRM soil moisture estimates are well correlated with in situ measurements and the variability of the measurements is higher than that of the ascending AMSR-LPRM soil moisture data. Fig. 9b shows 
that the most $R$ values of the descending orbit data are also greater than 0.8 , but the $S D V$ s values are above 1.0. This suggests that the descending AMSR-LPRM data has a larger variability than in situ measurements. The AMSR-JAXA and AMSR2 data show similar distributions and are more dispersive when compared to the AMSR-LPRM. In most cases, the $R$ values range from 0.65 to 0.85 and the $S D V$ s range from 0.8 to above 2.0 for both ascending and descending orbits data. These results indicate that the AMSR-JAXA and AMSR2 have larger variability than in situ measurements. This result is coincident with Figs. 7 and 8.

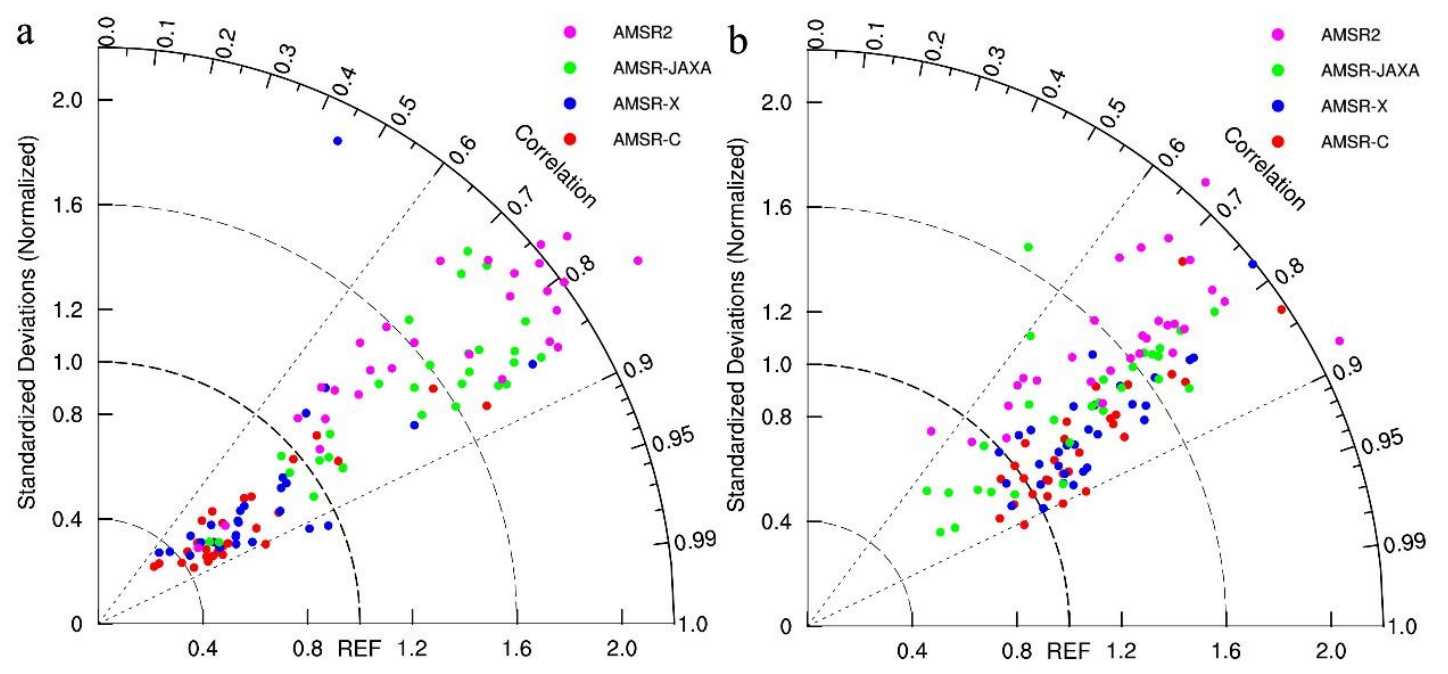

Fig. 9. Taylor diagrams of the comparisons between the soil moisture products and in situ measurements. a. ascending and $b$. descending orbit.

Because the spatial scales and the depths of model simulations $\left(0.1^{\circ}\right.$ and $\left.4.5 \mathrm{~cm}\right)$, AMSR-E and AMSR2 products $\left(0.25^{\circ}\right.$ and $\left.0-2 \mathrm{~cm}\right)$ and in situ measurements (point scale and $5 \mathrm{~cm}$ ) mismatched, direct absolute comparison may be inappropriate. To eliminate this issue, the normalized anomaly soil moistures were calculated (Figs. 10 and 11). For the AMSR-LPRM, the wet biases were reduced duo to the normalization. It shows better temporal variability patterns with in situ measurements despite some noises, when compared with direct absolute comparisons (Figs. $7 \&$ 8). However, the AMSR-JAXA and AMSR2 series show large biases, which are 
positive for unfrozen soil and negative for frozen soil. This is a reasonable result, because the variabilities of the two products are large (ie. large $\sigma_{s m}$ ). Overall, these results suggest that although there are significant biases for all the three remotely sensed soil moisture, they show potential monitoring soil moisture dynamics such as monitoring soil frozen-thaw process in this cold area. Compared to the remotely sensed data, the CLM4.0 modelled anomaly values were more consistent with in situ measurement series except for relative large biases during frozen periods.

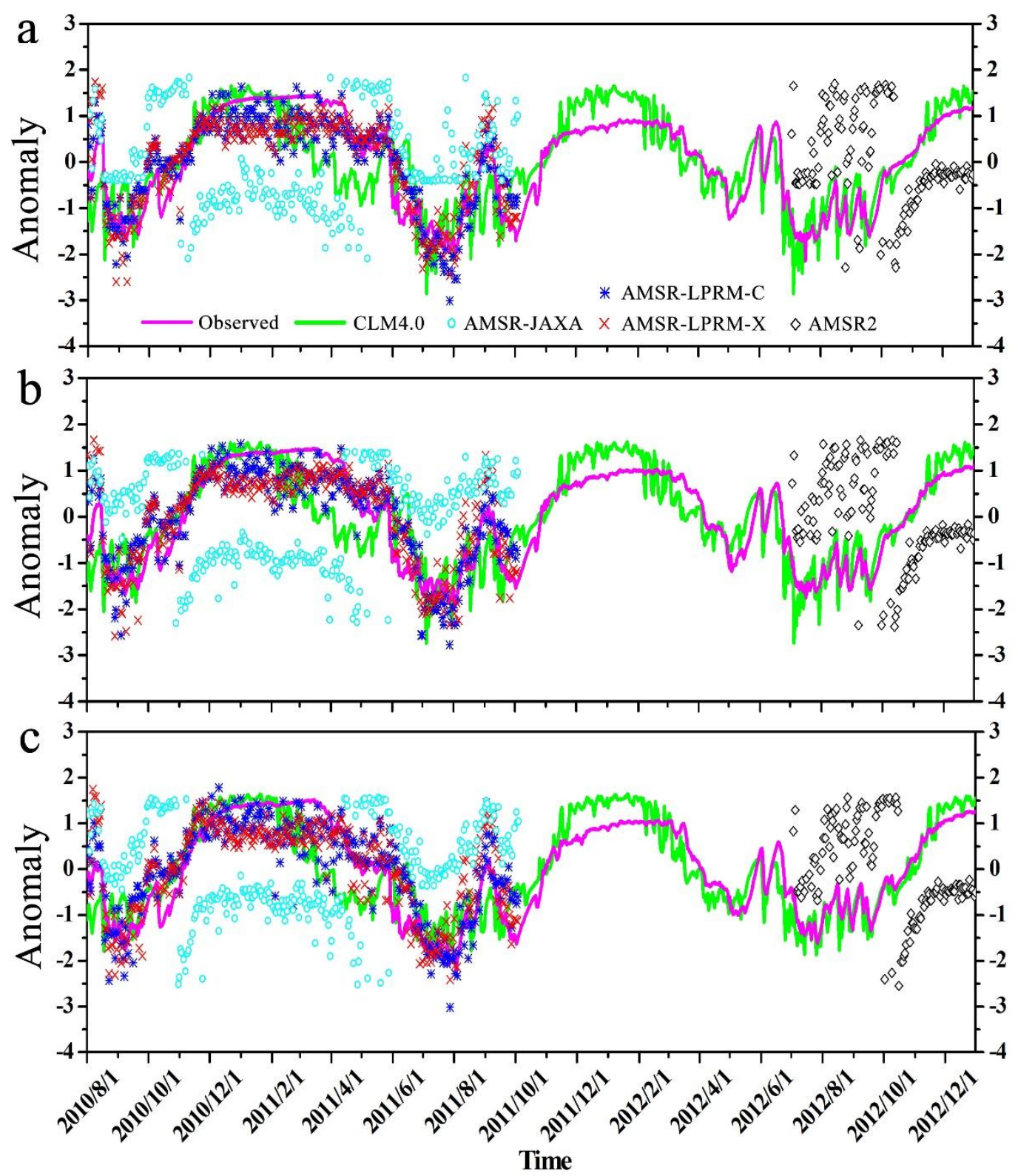


Fig. 10. Normalized anomaly of soil moisture time series at three scales: in situ measurements, the CLM4.0 model, the C- and X-band of AMSR-LPRM, the AMSR-JAXA and AMSR2 for ascending orbit. a. AMSR-E pixel; b. medium network; and c. large network.

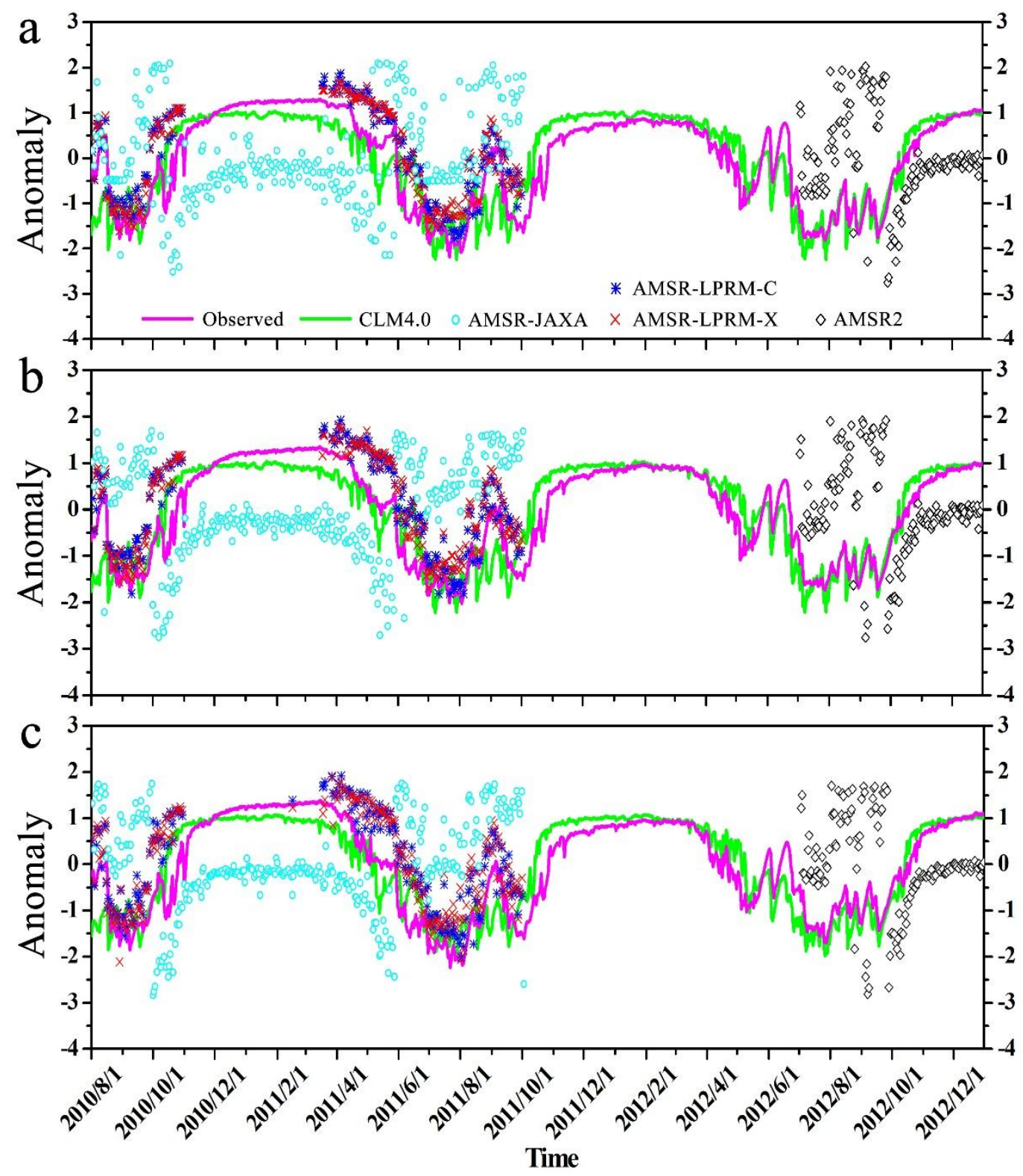

Fig. 11. Same as Fig.10, but for descending orbit.

\section{Discussion}

Soil moisture has very high spatiotemporal variability and is affected by precipitation, evapotranspiration, soil text and many other land surface characteristics. How to obtain soil 
moisture value in cold area such as the Tibetan Plateau is still a challenge work. Several researches have evaluated remote sensing and LSMs soil moisture in this high altitude and cold area, but in most of these studies models was run at a station scale and there were not inter-comparisons between the modeled and remotely-sensed soil moistures.

For the AMSR-LPRM product, the absolute comparisons between the AMSR-LPRM and in situ measurements showed that the AMSR-LPRM poorly retrieved soil moisture value in the TP area. The RMSE and BIAS values were significant larger than other products. Because the biomass, air mass and air moisture are low and the RFI is rare in the study area, we can further concluded that the LPRM algorithm fails to retrieve soil moisture value in the TP area. The AMSR-JAXA product performed better than the other remotely sensed products and captured frozen soil moisture value well. This result verifies previous studies that the JAXA algorithm performs well under light-vegetation conditions despite overestimation biases (Jackson et al., 2010; Kim et al., 2015). The AMSR2 data showed a similar performance to AMSR-JAXA for retrieving soil moisture values. Although it has a small negative average BIAS value, we cannot make a same conclusion to previous study (Kim et al., 2015; Wu et al., 2016) that the JAXA AMSR2 soil moisture are lower than in situ observations, because its very large variability for unfrozen soil. As the successor of AMSR-E, more research is needed to calibrate the AMSR2 soil moisture product and further align it to the AMSR-E sensor. Indeed, the uncertainties of the remotely sensed soil moisture products are caused by several factors synthesized. Except the most related algorithm factors (mean temperature, surface roughness, vegetation density and mean ground soil moisture) which have been fully discussed by Kim et al. (2015), following aspects should be considered. First, the differences of scales: as it is well known, the representativeness of in situ measurements 
at the remote sensing pixel scale is uncertain. Second, the differences in soil depths between remotely sensed soil moisture and observations: the remotely sensed data have a limited depth $(<$ $2 \mathrm{~cm}$ ), and the soil moisture observation at $5 \mathrm{~cm}$ depth is an average value for the top soil layer $(0-5 \mathrm{~cm})$. Furthermore, the RFI in the 0.4 to $11 \mathrm{GHz}$ spectrum is growing increase and its effect on the sensor X-band measurements is another uncertainty source for the remotely sensed soil moisture (National Academies Press, 2015). Consequently, it is concluded that there are still significant biases for all the three remote sensing soil moisture in the TP area and the retrieval algorithms need to be further improved.

The accuracy of LSM-modeled soil moistures largely depends on the quality of forcing data. Forcing the CLM4.0 model with a newly developed meteorological data set, we obtained high-resolution simulated soil moisture data. The absolute comparisons with in situ measurements show that the model captured soil moisture variability well in most time of the simulation years (mean RMSE $0.03 \mathrm{~m}^{3} \mathrm{~m}^{-3}$ ). However, there were still some uncertainties, including the uncertainties for frozen soil, the surface layers underestimations and deeper layers overestimations for unfrozen soil. Four aspects have been considered as the sources of these uncertainties. The first one is the parameterization schemes of the model: the frozen-thaw process is very strong in this area and the model parameterizations is still insufficient for representing these coupled processes. The second aspect is the accuracy of forcing and land surface property (soil texture, organic matter, vegetation condition and others) data sets: as these data were developed by interpolating observations and atmospheric predictions or derived from remote sensing observations, we cannot truly obtain the localized data. Furthermore, the scale difference is another error source: the modelled soil moisture is a grid $\left(0.1 \times 0.1^{\circ}\right)$ mean value and the in situ measurement is a plot 
value. Finally, the difference of soil layer depths between the model outputs and in situ measurements may also cause large errors even though interpolations have been conducted.

For different application purposes, the accuracy requirements of soil moisture products are different. For example, it is more to obtain a good representation of the temporal dynamics than the absolute soil moisture state for atmospheric studies and soil frozen-thaw monitoring (Rüdiger et al., 2009). Therefore, although the remote sensing data poorly estimated absolute soil moisture values in the TP area, they can be very important information for these fields. On the other hand, the absolute soil moisture value is very critical for hydrological, ecological and agricultural applications. Because of the large biases, the remote sensing soil moisture fails to meet these applications. For the modeled soil moisture, it can be used as an credible soil moisture approximate estimate, when lack of sufficient observations at large scale. Nevertheless, the LSMs modeled soil moisture still need to be improved by further enhancing the model parameterizations, input data sets and resolution.

\section{Conclusions}

In this study, we have compared three remotely sensed soil moisture data sets (AMSR-LPRM, AMSR-JAXA and AMSR2) and CLM4.0 modeled soil moisture using a soil moisture monitoring network located in the central Tibetan Plateau area. The results showed that both the three remotely sensed and the CLM4.0 modelled soil moisture reflected soil moisture dynamics well, with average R-values of 0.87 for the AMSR-LPRM, 0.85 for AMSR-JAXA, 0.8 for AMSR2 and 0.94 for CLM4.0 (5, 10, 20 and $40 \mathrm{~cm}$ depths), respectively. The AMSR-LPRM data tended to obviously overestimate soil moisture with BIAS ranging from $0.0907 \mathrm{~m}^{3} \mathrm{~m}^{-3}$ to $0.1456 \mathrm{~m}^{3} \mathrm{~m}^{-3}$ and the RMSE values were large (in average $0.1235 \mathrm{~m}^{3} \mathrm{~m}^{-3}$ ). The AMSR-JAXA performed better than 
the AMSR-LPRM and AMSR2, with small BIAS and RMSE values (0.0165 and $\left.0.0959 \mathrm{~m}^{3} \mathrm{~m}^{-3}\right)$. Especially, the AMSR-JAXA successfully retrieved soil moisture value under frozen condition. The AMSR2 showed similar performance to the AMSR-JAXA, but with slight larger BIAS and RMSE (in average 0.0242 and $0.1127 \mathrm{~m}^{3} \mathrm{~m}^{-3}$ ). Particularly, it showed a very large variability during unfrozen periods. Compared with the remotely sensed data, CLM4.0 produced better results, with low RMSE value of $0.03 \mathrm{~m}^{3} \mathrm{~m}^{-3}$. However, there are still some uncertainties for the modeled soil moisture. For example, it tended to slightly underestimate for the surface soil layers ( 5 and $10 \mathrm{~cm}$ ) with negative biases ranging from $-0.02 \mathrm{~m}^{3} \mathrm{~m}^{-3}$ to $-0.01 \mathrm{~m}^{3} \mathrm{~m}^{-3}$ and overestimated for deeper layers $\left(20\right.$ and $40 \mathrm{~cm}$ ) with biases ranging from $0.004 \mathrm{~m}^{3} \mathrm{~m}^{-3}$ to $0.03 \mathrm{~m}^{3} \mathrm{~m}^{-3}$. In general, we can draw the following conclusions:

1. All the three remotely sensed soil moisture products capture soil moisture dynamics well, but each of them has large biases for different conditions. The AMSR-LPRM systematically overestimates unfrozen soil moisture and fails to estimate frozen soil. The AMSR-JAXA shows the best performance than the others, but it has an overestimation for unfrozen soil. The AMSR2 shows potential to continue to provide long-term and large-scale soil moisture, but has a very large variability during unfrozen periods.

2. Driven by a reliable and high-resolution forcing data, the CLM4.0 model can reasonably capture soil moisture changes. Based on the evaluations, the modelled soil moistures may be used as a credible approximate estimate in absence of observations in the Tibetan Plateau area, despite some uncertainties such as a slight underestimation for the surface soil layers and relative poor estimates for frozen soil.

3. The remote sensing and model soil moistures show a good correlation with each other. This 
suggests the potential of using one of them to validate the other for the situation that lack of enough observations in the Tibetan Plateau area.

In summary, as many previous studies pointed out there is still much room for improving the remotely sensed soil moisture algorithms. Especially for the currently AMSR2 product more efforts are needed to calibrate the data and further align it to the AMSR-E sensor. For the LSMs simulated soil moisture, future work should focus on improving the model structures and parameterizations and reducing the uncertainties of the model inputs.

\section{Acknowledgments}

The authors would like to thank Drs. Kun Yang and Yingying Chen (Institute of Tibetan Plateau Research, Chinese Academy of Sciences) for providing the in situ SM data, and Yaoxian Yang (Institute of Atmospheric Physics, Chinese Academy of Sciences) for his help on data processing. This work was supported by the research grant (41271116) funded by the National Science Foundation of China, the research grant (2012ZD010) of the Key Project for the Strategic Science Plan in IGSNRR, CAS, China Postdoctoral Science Foundation (2015M571102) and the National Science Foundation of China (31500402). 


\section{References}

Albergel, C., de Rosnay, P., Gruhier, C., Muñoz-Sabater, J., Hasenauer, S., Isaksen, L., Kerr, Y. and Wagner, W., 2012. Evaluation of remotely sensed and modelled soil moisture products using global ground-based in situ observations. Remote Sensing of Environment, 118: 215-226. http://dx.doi.org/10.1016/j.rse.2011.11.017.

Boisserie, M. and Cocke, S., 2012. Development of a soil moisture analysis for subseasonal forecasting: Soil moisture validation. Journal of Geophysical Research: Atmospheres (1984-2012), 117(D10105). http://dx.doi.org/10.1029/2011JD015977.

Brocca, L., Hasenauer, S., Lacava, T., Melone, F., Moramarco, T., Wagner, W., Dorigo, W., Matgen, P., Martínez-Fernández, J. and Llorens, P., 2011. Soil moisture estimation through ASCAT and AMSR-E sensors: An intercomparison and validation study across Europe. Remote Sensing of Environment, 115(12): 3390-3408.

Brocca, L., Melone, F., Moramarco, T., Wagner, W. and Hasenauer, S., 2010. ASCAT soil wetness index validation through in situ and modeled soil moisture data in central Italy. Remote Sensing of Environment, 114(11): 2745-2755.

Champagne, C., McNairn, H. and Berg, A.A., 2011. Monitoring agricultural soil moisture extremes in Canada using passive microwave remote sensing. Remote Sensing of Environment, 115(10): 2434-2444.

Chen, B.L., Luo, S.Q., Lü, S.H. and Zhang, Y., 2014. Validation and comparison of the simulation at Zoigê station during freezing and thawing with land surface model CLM. Climatic Environment Research, 19(5): 649-658. (in Chinese)

Chen, Y., Yang, K., Qin, J., Zhao, L., Tang, W. and Han, M., 2013. Evaluation of AMSR - E retrievals and GLDAS simulations against observations of a soil moisture network on the central Tibetan Plateau. Journal of Geophysical Research: Atmospheres, 118(10): $4466-4475$.

Chen, Y., Yang, K., Tang, W., Qin, J. and Zhao, L., 2012. Parameterizing soil organic carbon's impacts on soil porosity and thermal parameters for Eastern Tibet grasslands. Science China Earth Sciences, 55(6): 1001-1011.

Cho, E., Choi, M. and Wagner, W., 2015a. An assessment of remotely sensed surface and root 
zone soil moisture through active and passive sensors in northeast Asia. Remote Sensing of Environment, 160: 166-179.

Cho, E., Moon, H. and Choi, M., 2015b. First Assessment of the Advanced Microwave Scanning Radiometer 2 (AMSR2) Soil Moisture Contents in Northeast Asia. Journal of the Meteorological Society of Japan, 93(1): 117-129.

Choi, M., 2012. Evaluation of multiple surface soil moisture for Korean regional flux monitoring network sites: Advanced Microwave Scanning Radiometer E, land surface model, and ground measurements. Hydrological Processes, 26(4): 597-603.

Coopersmith, E.J., Cosh, M.H., Bindlish, R. and Bell, J., 2015. Comparing AMSR-E soil moisture estimates to the extended record of the U.S. Climate Reference Network (USCRN). Advances in Water Resources, 85: 79-85.

Crawford, T.M. and Duchon, C.E., 1999. An improved parameterization for estimating effective atmospheric emissivity for use in calculating daytime downwelling longwave radiation. Journal of Applied Meteorology, 38(4): 474-480.

de Jeu, R.A.M., Holmes, T.R.H., Parinussa, R.M. and Owe, M., 2014. A spatially coherent global soil moisture product with improved temporal resolution. Journal of Hydrology, 516: 284-296.

Dirmeyer, P.A., Gao, X., Zhao, M., Guo, Z., Oki, T. and Hanasaki, N., 2006. GSWP-2: Multimodel analysis and implications for our perception of the land surface. Bulletin of the American Meteorological Society, 87(10): 1381-1397.

Dirmeyer, P.A., Guo, Z.C. and Gao, X., 2004. Comparison, validation, and transferability of eight multiyear global soil wetness products. Journal of Hydrometeorology, 5(6): 1011-1033.

Draper, C.S., Walker, J.P., Steinle, P.J., de Jeu, R.A. and Holmes, T.R., 2009. An evaluation of AMSR-E derived soil moisture over Australia. Remote Sensing of Environment, 113(4): 703-710.

Fujii, H., Koike, T. and Imaoka, K., 2009. Improvement of the AMSR-E algorithm for soil moisture estimation by introducing a fractional vegetation coverage dataset derived from MODIS data. Journal of the Remote Sensing Society of Japan, 29(1): 282-292.

Gao, Y., Li, K., Chen, F., Jiang, Y. and Lu, C., 2015. Assessing and improving Noah-MP land model simulations for the central Tibetan Plateau. Journal of Geophysical Research: 
Atmospheres, 120(18): 9258-9278.

Georgakakos, K.P. and Carpenter, T.M., 2006. Potential value of operationally available and spatially distributed ensemble soil water estimates for agriculture. Journal of Hydrology, 328: 177-191. http://dx.doi.org/10.1016/j.jhydrol.2005.12.018.

Gevaert, A.I., Parinussa, R.M., Renzullo, L.J., van Dijk, A.I.J.M. and de Jeu, R.A.M., 2016. Spatio-temporal evaluation of resolution enhancement for passive microwave soil moisture and vegetation optical depth. International Journal of Applied Earth Observation and Geoinformation, 45: 235-244.

Gruhier, C., De Rosnay, P., Hasenauer, S., Holmes, T., De Jeu, R., Kerr, Y., Mougin, E., Njoku, E., Timouk, F. and Wagner, W., 2010. Soil moisture active and passive microwave products: intercomparison and evaluation over a Sahelian site. Hydrology and Earth System Sciences, 14(1): 141-156.

Gruhier, C., De Rosnay, P., Kerr, Y., Mougin, E., Ceschia, E., Calvet, J.C. and Richaume, P., 2008. Evaluation of AMSR-E soil moisture product based on ground measurements over temperate and semi-arid regions. Geophysical Research Letters, 35(L10405). http://dx.doi.org/10.1029/2008GL033330.

Guo, D. and Wang, H., 2013. Simulation of permafrost and seasonally frozen ground conditions on the Tibetan Plateau, 1981-2010. Journal of Geophysical Research: Atmospheres, 118(11): 5216-5230.

Guo, Z., Dirmeyer, P.A., Hu, Z.Z., Gao, X. and Zhao, M., 2006. Evaluation of the Second Global Soil Wetness Project soil moisture simulations: 2. Sensitivity to external meteorological forcing. Journal of Geophysical Research: Atmospheres (1984-2012), 111(D22S03). http://dx.doi.org/10.1029/2006JD007845

Hain, C.R., Crow, W.T., Mecikalski, J.R., Anderson, M.C. and Holmes, T., 2011. An intercomparison of available soil moisture estimates from thermal infrared and passive microwave remote sensing and land surface modeling. Journal of Geophysical Research-Atmospheres, 116(D15107). http://dx.doi.org/10.1029/2011JD015633.

Huffman, G.J., Bolvin, D.T., Nelkin, E.J., Wolff, D.B., Adler, R.F., Gu, G., Hong, Y., Bowman, K.P. and Stocker, E.F., 2007. The TRMM multisatellite precipitation analysis (TMPA): Quasi-global, multiyear, combined-sensor precipitation estimates at fine scales. Journal of Page 35 
Hydrometeorology, 8(1): 38-55.

Imaoka, K., Kachi, M., Kasahara, M., Ito, N., Nakagawa, K. and Oki, T., 2010. Instrument performance and calibration of AMSR-E and AMSR2. International Archives of the Photogrammetry, Remote Sensing and Spatial Information Science, 38(8): 13-18.

Jackson, T.J., 1993. III. Measuring surface soil moisture using passive microwave remote sensing. Hydrological processes, 7(2): 139-152.

Jackson, T.J., Cosh, M.H., Bindlish, R., Starks, P.J., Bosch, D.D., Seyfried, M., Goodrich, D.C., Moran, M.S. and Du, J., 2010. Validation of advanced microwave scanning radiometer soil moisture products. Geoscience and Remote Sensing, IEEE Transactions on, 48(12): $4256-4272$.

Jajarmizadeh, M., Harun, S.b., Shahid, S., Akib, S. and Salarpour, M., 2014. Impact of Direct Soil Moisture and Revised Soil Moisture Index Methods on Hydrologic Predictions in an Arid Climate. Advances in Meteorology, 2014: 8. http://dx.doi.org/10.1155/2014/156172.

Kachi, M., Hori, M., Maeda, T. and Imaoka, K., 2014. Status of validation of AMSR2 on board the GCOM-W1 satellite. Geoscience and Remote Sensing Symposium (IGARSS), 2014 IEEE International, 110-113.

Kang, S., Xu, Y., You, Q., Flügel, W.-A., Pepin, N. and Yao, T., 2010. Review of climate and cryospheric change in the Tibetan Plateau. Environmental Research Letters, 5(1): 015101. http://dx.doi.org/10.1088/1748-9326/5/1/015101.

Kerr, Y.H., Waldteufel, P., Wigneron, J.P., Font, J. and Berger, M., 2001. Soil moisture retrieval from space: The Soil Moisture and Ocean Salinity (SMOS) mission. Geoscience and Remote Sensing, IEEE Transactions on, 39(8): 1729-1735.

Kim, S., Liu, Y.Y., Johnson, F.M., Parinussa, R.M. and Sharma, A., 2015. A global comparison of alternate AMSR2 soil moisture products: Why do they differ? Remote Sensing of Environment, 161: 43-62.

Kim, Y. and Wang, G., 2012. Soil moisture - vegetation - precipitation feedback over North America: Its sensitivity to soil moisture climatology. Journal of Geophysical Research: Atmospheres (1984-2012), 117(D18). http://dx.doi.org/10.1029/2012JD017584.

Koike, T., Nakamura, Y., Kaihotsu, I., Davva, N., Matsuura, N., Tamagawa, K., and Fujii, H., 2004. Development of an Advanced Microwave Scanning Radiometer (AMSR-E) algorithm of 
soil moisture and vegetation water content. Annual Journal of Hydraulic Engineering, JSCE, 48: $217-222$.

Koster, R.D., Mahanama, S.P., Livneh, B., Lettenmaier, D.P. and Reichle, R.H., 2010. Skill in streamflow forecasts derived from large-scale estimates of soil moisture and snow. Nature Geoscience, 3(9): 613-616.

Lai, X., Wen, J., Ceng, S.X., Song, H.Q., Tian, H., Shi, X.K., He, Y. and Huang, X., 2014. Numerical simulation and evaluation study of soil moisture over China by using CLM4.0 model. Chinese Journal of Atmospheric Sciences, 38(3): 499-512. (in Chinese)

Lawrence, D.M., Oleson, K.W., Flanner, M.G., Thornton, P.E., Swenson, S.C., Lawrence, P.J., Zeng, X., Yang, Z.-L., Levis, S., Sakaguchi, K., Bonan, G.B. and Slater, A.G., 2011. Parameterization improvements and functional and structural advances in Version 4 of the Community Land Model. Journal of Advances in Modeling Earth Systems, 3(M03001). http://dx.doi.org/10.1029/2011MS000045.

Li, Y., Liu, X. and Li, W., 2012. Numerical simulation of land surface process at different underlying surfaces in Tibetan Plateau. Climatic Environment Research, 31(3): 581-591. (in Chinese)

National Academies Press, 2015. A Strategy for Active Remote Sensing Amid Increased Demand for Radio Spectrum. Washington, DC., The National Academies Press, Chapter 8: 161-188. http://dx.doi.org/10.17226/21729.

Njoku, E.G. and Chan, S.K., 2006. Vegetation and surface roughness effects on AMSR-E land observations. Remote Sensing of Environment, 100(2): 190-199.

Njoku, E.G., Jackson, T.J., Lakshmi, V. and Chan, T.K., 2003. Soil moisture retrieval from AMSR-E. IEEE Transactions on Geoscience and Remote Sensing, 41(2): 215-229.

Ochsner, T.E., Cosh, M.H., Cuenca, R.H., Dorigo, W.A., Draper, C.S., Hagimoto, Y., Kerr, Y.H., Larson, K.M., Njoku, E.G., Small, E.E. and Zreda, M., 2013. State of the Art in Large-Scale Soil Moisture Monitoring. Soil Science Society of America Journal, 77(6): 1888-1919.

Oleson, K.W., Lawrence, D.M., Gordon, B., Flanner, M.G., Kluzek, E., Peter, J., Levis, S., Swenson, S.C., Thornton, E. and Feddema, J., 2010. Technical description of version 4.0 of the Community Land Model (CLM). NCAR, Boulder.

Owe, M., de Jeu, R. and Holmes, T., 2008. Multisensor historical climatology of satellite-derived 
global land surface moisture. Journal of Geophysical Research: Earth Surface (2003-2012), 113(F01002). http://dx.doi.org/10.1029/2007JF000769.

Pan, M., Sahoo, A.K. and Wood, E.F., 2014. Improving soil moisture retrievals from a physically-based radiative transfer model. Remote Sensing of Environment, 140(1): $130-140$

Parinussa, R.M., Holmes, T.R.H., Wanders, N., Dorigo, W.A. and de Jeu, R.A.M., 2015. A Preliminary Study toward Consistent Soil Moisture from AMSR2. Journal of Hydrometeorology, 16(2): 932-947.

Pinker, R.T. and Laszlo, I., 1992. Modeling surface solar irradiance for satellite applications on as global scale. Journal of Applied Meteorology, 31(2): 194-211.

Reichle, R.H., Koster, R.D., Liu, P., Mahanama, S.P.P., Njoku, E.G. and Owe, M., 2007. Comparison and assimilation of global soil moisture retrievals from the Advanced Microwave Scanning Radiometer for the Earth Observing System (AMSR-E) and the Scanning Multichannel Microwave Radiometer (SMMR). Journal of Geophysical Research Atmospheres, 112(D9): 139-155.

Rodell, M., Houser, P., Jambor, U.e.a., Gottschalck, J., Mitchell, K., Meng, C., Arsenault, K., Cosgrove, B., Radakovich, J. and Bosilovich, M., 2004. The global land data assimilation system. Bulletin of the American Meteorological Society, 85(3): 381-394.

Rüdiger, C., Calvet, J-C., Gruhier, C., Holmes, T.R., De Jeu, R.A. and Wagner, W., 2009. An intercomparison of ERS-Scat and AMSR-E soil moisture observations with model simulations over France. Journal of Hydrometeorology, 10(2): 431-447.

Seneviratne, S.I., Corti, T., Davin, E.L., Hirschi, M., Jaeger, E.B., Lehner, I., Orlowsky, B. and Teuling, A.J., 2010. Investigating soil moisture-climate interactions in a changing climate: A review. Earth-Science Reviews, 99(3): 125-161.

Sheffield, J., Goteti, G. and Wood, E.F., 2006. Development of a 50-year high-resolution global dataset of meteorological forcings for land surface modeling. Journal of Climate, 19(13): 3088-3111.

Singh, R.S., Reager, J.T., Miller, N.L. and Famiglietti, J.S., 2015. Toward hyper-resolution land-surface modeling: The effects of fine-scale topography and soil texture on CLM4.0 simulations over the Southwestern US. Water Resources Research, 51(4): 2648-2667. 
Su, C.-H., Ryu, D., Young, R.I., Western, A.W. and Wagner, W., 2013. Inter-comparison of microwave satellite soil moisture retrievals over the Murrumbidgee Basin, southeast Australia. Remote Sensing of Environment, 134: 1-11.

Su, Z., Wen, J., Dente, L., Velde, R., Wang, L., Ma, Y., Yang, K. and Hu, Z., 2011. The Tibetan Plateau observatory of plateau scale soil moisture and soil temperature (Tibet-Obs) for quantifying uncertainties in coarse resolution satellite and model products. Hydrology and Earth System Sciences, 15(7): 2303-2316.

Swenson, S., Lawrence, D. and Lee, H., 2012. Improved simulation of the terrestrial hydrological cycle in permafrost regions by the Community Land Model. Journal of Advances in Modeling Earth Systems, 4(M08002). http://dx.doi.org/10.1029/2012MS000165.

Taylor, K.E., 2001. Summarizing multiple aspects of model performance in a single diagram. Journal of Geophysical Research: Atmospheres (1984-2012), 106(D7): 7183-7192.

Wagner, W., Naeimi, V., Scipal, K., de Jeu, R. and Martínez-Fernández, J., 2007. Soil moisture from operational meteorological satellites. Hydrogeology Journal, 15(1): 121-131.

Wei, J., Dirmeyer, P.A. and Guo, Z., 2008. Sensitivities of soil wetness simulation to uncertainties in precipitation and radiation. Geophysical Research Letters, 35(L15703). http://dx.doi.org/10.1029/2008GL034494.

Wu, Q., Liu, H., Wang, L. and Deng, C., 2016. Evaluation of AMSR2 soil moisture products over the contiguous United States using in situ data from the International Soil Moisture Network. International Journal of Applied Earth Observation and Geoinformation, 45: 187-199.

Xia, K., Luo, Y. and Li, W.P., 2011. Simulation of freezing and melting of soil on the northeast Tibetan Plateau. Chinese Science Bulletin, 56(22): 1828-1838.

Xiao, Y., Zhao, L., Dai, Y.J., Li, R., Pang, Q.Q. and Yao, J.M., 2013. Representing permafrost properties in CoLM for the Qinghai-Xizang (Tibetan) Plateau. Cold Regions Science and Technology, 87: 68-77. http://dx.doi.org/10.1016/j.coldregions.2012.12.004.

Yang, K., Chen, Y.Y. and Qin, J., 2009. Some practical notes on the land surface modeling in the Tibetan Plateau. Hydrol. Earth Syst. Sci., 13(5): 687-701.

Yang, K., He, J., Tang, W., Qin, J. and Cheng, C.C., 2010. On downward shortwave and longwave radiations over high altitude regions: Observation and modeling in the Tibetan Plateau. Agricultural and Forest Meteorology, 150(1): 38-46. 
Yang, K., Qin, J., Zhao, L., Chen, Y., Tang, W., Han, M., Chen, Z., Lv, N., Ding, B. and Wu, H., 2013. A multiscale soil moisture and freeze-thaw monitoring network on the third pole. Bulletin of the American Meteorological Society, 94(12): 1907-1916.

Yang, K., Wu, H., Qin, J., Lin, C., Tang, W. and Chen, Y., 2014. Recent climate changes over the Tibetan Plateau and their impacts on energy and water cycle: A review. Global and Planetary Change, 112: 79-91. http://dx.doi.org/10.1016/j.gloplacha.2013.12.001.

Yatagai, A., Arakawa, O., Kamiguchi, K., Kawamoto, H., Nodzu, M.I. and Hamada, A., 2009. A 44-Year Daily Gridded Precipitation Dataset for Asia Based on a Dense Network of Rain Gauges. Sola, 5: 137-140. http://dx.doi.org/10.2151/sola.2009-035.

Zeng, J., Li, Z., Chen, Q., Bi, H., Qiu, J. and Zou, P., 2015. Evaluation of remotely sensed and reanalysis soil moisture products over the Tibetan Plateau using in-situ observations. Remote Sensing of Environment, 163: 91-110. http://dx.doi.org/10.1016/j.rse.2015.03.008.

Zhang, X-J., Tang, Q., Pan, M. and Tang, Y., 2014. A long-term land surface hydrologic fluxes and states dataset for China. Journal of Hydrometeorology, 15(5): 2067-2084. 